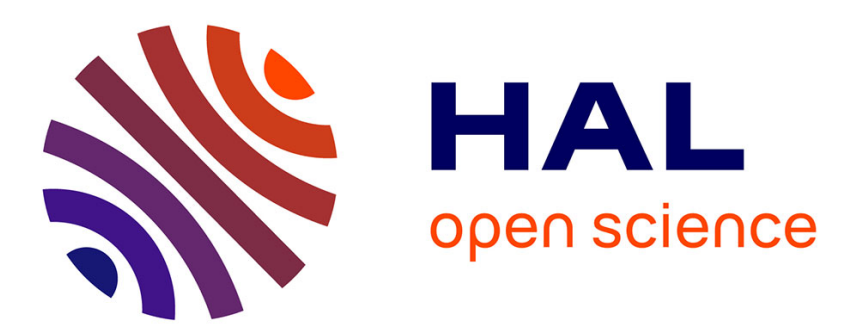

\title{
Is it possible to conserve electric charge without conserving separately baryonic number and leptonic number?
}

\author{
U.C. Tsan
}

\section{To cite this version:}

U.C. Tsan. Is it possible to conserve electric charge without conserving separately baryonic number and leptonic number?. International Journal of Modern Physics E, 2008, 17, pp.1591-1603. 10.1142/S021830130801060X . in2p3-00221298

HAL Id: in2p3-00221298

https://hal.in2p3.fr/in2p3-00221298

Submitted on 28 Jan 2008

HAL is a multi-disciplinary open access archive for the deposit and dissemination of scientific research documents, whether they are published or not. The documents may come from teaching and research institutions in France or abroad, or from public or private research centers.
L'archive ouverte pluridisciplinaire HAL, est destinée au dépôt et à la diffusion de documents scientifiques de niveau recherche, publiés ou non, émanant des établissements d'enseignement et de recherche français ou étrangers, des laboratoires publics ou privés. 


\section{IS IT POSSIBLE TO CONSERVE ELECTRIC CHARGE WITHOUT CONSERVING SEPARATELY BARYONIC NUMBER AND LEPTONIC NUMBER?}

\section{TSAN UNG CHAN}

Laboratoire de Physique Subatomique et de Cosmologie,

Université Joseph Fourier Grenoble 1, CNRS/IN2P3, Institut Polytechnique de Grenoble, 53 rue des Martyrs, 38026 Grenoble Cedex, France

tsan@1psc.in2p3.fr

Charges which are sources of fields must be universally conserved. Any quantity which is proved to be violated in certain circumstance cannot be a source of field. To account for the asymmetry of our universe baryon number A (or/and lepton number L) has to be violated thus A cannot be a charge. We speculate a new interaction MC with (A-L) as charge and $\mathrm{Z}^{*}$ as messenger. Conservation of (A-L) instead of (3A-L) suggested by Sakharov was deduced on one hand from observational fact (our universe is both material and neutral) and on the other hand from the generalized Gell-Mann and Nishijima formula. Conservation of (A-L) forbids neutrinoless double beta decay and neutron antineutron oscillations. The union of four interactions electromagnetism, $\mathrm{MC}$ interaction, weak interaction and strong interaction considered as the product $\mathrm{U}(1) \times \mathrm{U}(1) \times \mathrm{SU}(2) \times \mathrm{SU}(3)$ would account for available experimental and observational data. Observation of processes violating baryon number would be of great interest to falsify this suggestion.

Key-words: Universal conservation laws of energy, linear and angular momentum, CPT, Q, BAL and TF. 


\section{Introduction}

According to the Standard Model there are two kinds of matter elementary particles: three families of quarks bearing colour charge which are sensitive to strong interaction and three families of leptons not bearing colour charge which are not sensitive to strong interaction. Three interactions namely electromagnetism, strong interaction and weak interaction are at the origin of any microscopic process occurring in nature and in laboratories. Ultimately they are due to the exchange of messengers between two charged particles. Messenger of electromagnetism is photon, messengers of weak interaction are $\mathrm{W}$ and $\mathrm{Z}$ and messengers of strong force are gluons. We remark that each interaction has at least a neutral messenger which plays the role of neutral element. The exchange between two charged particles of this neutral messenger does not modify their internal characteristics.

Conservation principles learned through experiences play a fundamental role in the understanding of processes observed in experiment and processes never observed in experiment. Each interaction has its own absolute conservation laws (invariance of a defined quantity) e.g. any process governed by this interaction fulfils without exception all these laws. To be universal an absolute conservation law has to be common to all known and yet unknown interactions. An absolute conservation law valid in an interaction and not valid in another is not a universal conservation law. For example individual flavours are strictly conserved in electromagnetism and strong interaction but are not conserved in weak interaction. Conservation of individual flavours is thus not a universal law. A postulated process to be possible has to obey strictly all known and not yet known universal conservation laws.

\section{Space-time symmetries and internal symmetries}

There are two kinds of universal conservation laws: those related to space-time symmetries and those not related to space-time symmetries. Conservation of energy, linear momentum and angular momentum are deeply related to space-time symmetries. They are connected to homogeneity of time and homogeneity and isotropy of space. These conservation laws are fulfilled by all known interactions, namely gravitation, electromagnetism, strong interaction and weak interaction. If there is another not yet known interaction, it should also obey all these laws.

Conservation of electric charge $\mathrm{Q}$ belongs in contrast to the material type and is related to internal symmetries. Q having dynamical roles is believed to be very fundamental. Invariance of electric charge Q must be universal e.g. fulfilled by any known or yet unknown interaction because it is the source of electromagnetism. It means that 'whatever happens in 
any reaction the total amount of charge on one side has to balance with the amount on the other side'. (1). However the conservation of electric charge Q is only necessary but it is clearly not sufficient. So in classical physics to account for observation the conservation of mass which is considered as the conservation of matter is also required. Apparently all processes taking place in classical physics could be accounted for by the above conservation laws (the conservation of energy, of linear momentum, of angular momentum, the conservation of $\mathrm{Q}$ and of mass.) (2)

Before Einstein, mass was considered to be a good measure of matter, matter having mass. Intuitively conservation of mass or conservation of matter represented the same reality. Classical physics must obey the principle of matter conservation which states that matter cannot be created or destroyed but can only be changed from one form to another. Counting is a natural and efficient way to make sure that an entity is conserved. An entity is conserved if the number of this entity is the same in the initial channel and in the final channel. For example chemical equations basically and naturally express the conservation of matter by a simple counting rule. The number of atoms of a particular species in the reactants must equal the number of those atoms in the products (stoichiometric balance). A chemical equation must be balanced because atoms are neither created nor destroyed according to the principle of matter conservation. The identities of the reactants and the products remain unchanged in a balanced equation.

In fact conservation of mass in classical physics is a direct consequence of conservation of atoms in chemical equations (only particles of the first family are involved), mass of an atom being considered to be constant whatever its combination with other atoms to form a molecule is. In reality mass is only one form of energy. In classical physics this form of energy which remains approximately constant is not included in equation stating the conservation of energy. In contrast in nuclear physics and particle physics mass clearly is not a constant but energy remains constant and mass should be included in the expression of energy. Conservation of matter is no more reflected by conservation of mass but reflected by conservation of baryon number A and lepton number L. We remark that conservation of atoms is indeed a particular case of conservation of A (particles sensitive to strong interaction) and $\mathrm{L}$ (particles insensitive to strong interaction). All known microscopic processes conserve these numbers. In contrast flavour numbers (upness, downess, strangeness ...) are conserved in processes governed by electromagnetism and strong interaction while they are not conserved in weak interaction.

\section{Interactions, charges and messengers}


Each interaction is characterized by its charges and its messengers: electric charge Q and $\gamma$ for electromagnetism, colour charges and gluons for strong interaction, weak charges and $\mathrm{W}$ and $\mathrm{Z}$ for weak interaction. If there is another interaction it should be characterized by another charge and another messengers. And it could have less conservation laws than the other interactions but it has to conserve anyway all known charges (electric, colour and weak charges). Conservation of electric charge is universal, conservation of colour charge is also universal (all observed processes are colourless), conservation of weak charge seems to be hidden behind the simultaneous conservation of $\mathrm{A}, \mathrm{L}$ and $\mathrm{Q}$ which automatically conserve $\mathrm{TH}$ defined as the sum of all hadronic and leptonic flavours. We could interpret TF as weak charge. We remark that colour charge is completely independent of Q while A, L and flavours are related to $Q$ through the generalized Gell-Mann and Nishijima formula. (3)

\section{General relation between $Q, A, L$, hadronic and leptonic flavours}

For any particle (hadrons, leptons, messengers) we have the following relations

$$
\begin{aligned}
& \mathrm{Q}=\frac{A}{2}-\frac{L}{2}+\frac{T H F}{2}+\frac{T L F}{2} \\
& \text { or } \quad \mathrm{Q}=\frac{B A L}{2}+\frac{T F}{2}
\end{aligned}
$$

with $\mathrm{BAL}=\mathrm{A}-\mathrm{L}$ and $\mathrm{TF}=\mathrm{THF}+\mathrm{TLF}$

where $\mathrm{A}$ is the baryon number, $\mathrm{L}$ is the lepton number, THF is the total hadronic flavour, TLF is the total leptonic flavour and TF the total flavour.

$$
\mathrm{THF}=\mathrm{D}+\mathrm{U}+\mathrm{S}+\mathrm{C}+\mathrm{B}+\mathrm{T} \text { and } \mathrm{TLF}=\mathrm{D}_{1}+\mathrm{U}_{1}+\mathrm{S}_{1}+\mathrm{C}_{1}+\mathrm{B}_{1}+\mathrm{T}_{1} .
$$

Baryon number A characterizes matter particles sensitive to strong interaction and lepton number $\mathrm{L}$ characterizes matter particles insensitive to strong interaction. It is then natural to group them together into BAL as we group flavours terms into $\mathrm{TF}$. We note that flavour numbers of quarks (baryonic matter) and flavour numbers of leptons (leptonic matter) are either positive or negative ( 1 or -1$)$. We point out that $\mathrm{Q}$ of hadrons depends on A while $\mathrm{Q}$ of leptons depends on (-L). BAL is defined as baryon number minus lepton number (or baryon plus antilepton number). BAL is positive for baryonic matter particles sensitive to strong interaction and negative for leptonic matter particles insensitive to strong interaction. Any elementary or composite particle should be defined by these two numbers and not just by the algebraic value of $\mathrm{Q}$ which is equal to the half sum of these two independent and irreducible quantities. BAL is reduced to A for hadronic particles, to (-L) for leptons and to 0 for messengers ( $\gamma, \mathrm{W}, \mathrm{Z}$ and gluons). We suggested that weak charge could be identified to TF which is defined as the sum of all hadronic and leptonic flavours. Due to the relation 
between $\mathrm{Q}, \mathrm{A}, \mathrm{L}$ and flavours, conservation of $\mathrm{A}, \mathrm{L}$ and $\mathrm{Q}$ imply conservation of TF. As the two other charges conservation of TF should be universal.

\section{A and $L$ are strictly conserved in the Standard Model}

A, L, TF, Q and colour charge are separately conserved in processes governed by electromagnetism, strong interaction and weak interaction of the Standard Model. If there is no interaction other than these interactions then all these quantities should be considered to be universally conserved. However if there is sound reason to believe that electric charge, colour charge and weak charge are universally conserved since they are source of fields, conservation of $\mathrm{A}$ and conservation of $\mathrm{L}$ are only empirical rules and apparently have no theoretical justification. One tried to justify theoretically the universal conservation of $\mathrm{A}$ by assuming the existence of a baryonic force. But all efforts to search for baryonic force have so far failed. (4) Even if there is no univocal evidence of A violation in any experimentally observed microscopic process, one could not exclude that A could be violated in certain circumstances without violating any known universal laws of conservation such as electric charge, colour charge and weak charge. There are thus many attempts to search for theoretically and experimentally possible violation of $\mathrm{A}$.

Many unified theories like SU(5) suppose that A could be violated but (A-L) has to be universally conserved. Proton decay is predicted in this framework. However 'no examples of proton decay have been observed; depending on the decay mode, the proton lifetime is inferred to be longer than $10^{31}$ to $10^{33}$ years, surpassing the lifetime predicted by the SU(5) theory'. (5) There is thus not yet univocal positive signal of the violation of baryon number.

Neutron-antineutron oscillation if it exists would apparently violate A conservation while conserving electric charge and leptonic number since neutron and antineutron are both neutral. These oscillations were sought for on a beam of cold neutrons. Until now there is no positive signal of these oscillations. (6)

Existence of neutrinoless double beta decay would signal the violation of leptonic number (non conservation of leptonic matter) while conserving electric charge and baryonic number. Despite gigantic efforts to improve sensitivity and to diminish drastically the background there is still no univocal signal of this process.(7-12)

An interesting question would then be: is it necessary to suppose that there exists a process (governed by an interaction other than those of the Standard Model) which would violate the conservation of baryonic number or/and leptonic number? After all, all observed processes do conserve both $\mathrm{A}$ and $\mathrm{L}$ and all attempts to put into evidence any violation of $\mathrm{A}$ or/and L have so far only led to limits. 


\section{Indirect evidence of A violation: asymmetry of our universe}

Conservation of matter (reflected by the conservation of A and the conservation of $\mathrm{L}$ ) seems to be a reasonable prejudice valid for all so far observed processes. However, paradoxically this prejudice is in perfect contradiction with the existence of our universe. In effect contrary to the initial conjecture of Dirac, our universe is not half material and half antimaterial. It is entirely made up of matter particles such as protons, neutrons and electrons. It could thus not be the outcome of materialization which always creates an entangled pair of matter particle and antimatter particle via one interaction of the Standard Model with the absolute conservation of baryonic number and leptonic number. In this case any matter particle should have been created with its antimatter particle counterpart. We remark that a matter particle always annihilates together with its corresponding antiparticle and vice-versa. A or L remains constant after materialization or annihilation. If at the very origin there was only energy, A and L should be zero and should remain zero if there were only materialization and annihilation. The fact that our Universe is made of matter particles and is neutral is thus a double message of nature to be simultaneously deciphered. It points to the possible existence in the past of a hypothetical interaction violating A and simultaneously conserving electric charge, colour charge and weak charge. This novel interaction should have novel charge and messengers. And conversely its conjectured charge should be conserved in all known interactions.

\section{New interaction with less conservation laws}

Weak interactions conserve only A, L, Q (and colour Q) while strong interaction and electromagnetism conserve in addition individual flavours. If a combination of $\mathrm{A}$ and $\mathrm{L}$ were a charge it would be conserved by any interaction of the Standard Model since the separate conservations of A and L conserve automatically a combination of A and L. It is completely analogous to the fact that weak interactions conserve only TF instead of conserving every flavour number as in strong interaction and electromagnetism.

There is a priori a great number of possibilities. Sakharov (13) suggested that this combination was (3A-L) supposing that quarks and leptons are on the same footing. (A-L) is on the contrary the basis of many unified theories like SU(5). Is there any sound argument in favour of one particular combination?

In materialization the counterpart of proton in pair production is antiproton $(\Delta \mathrm{A}=0$ and $\Delta \mathrm{L}=0)$ and the counterpart of electron in pair production is antielectron $(\Delta \mathrm{A}=0$ and $\Delta \mathrm{L}=0)$. The only way to balance the positive charge of a proton with another material particle is indeed to create at the same time an electron. Material and neutral universe suggests thus the 
creation of an entangled pair of proton electron. Simultaneous violation of $\mathrm{A}$ and $\mathrm{L}(\Delta \mathrm{A}=$ $\Delta \mathrm{L}=1$ ) would be a possible solution. It corresponds to the conservation of (A-L) and $\mathrm{Q}$.

Curiously scrutiny of equation (1) leads also to the conclusion that to be invariant the combination should be (A-L) (2). A hypothetical interaction MC (matter creation) would have a charge defined by $(A-L)$ and would admit a messenger $Z *$ defined by $(B A L=0, T F=0, Q=0$ and colour charge $=0$ ). We verify immediately that BAL is conserved in interactions of the Standard Model.

\section{Deductions from (A-L) universal conservation law}

Any process conserving separately A and L conserves automatically the conservation of (A-L). There is no contradiction to consider (A-L) as a charge of an interaction.

$\mathrm{MC}$ interaction could admit proton decay with the following selection rules: $(\Delta(\mathrm{A}-\mathrm{L})=$ 0 with $\Delta \mathrm{A}=\Delta \mathrm{L}=-1),(\Delta \mathrm{Q}=0),(\Delta \mathrm{TF}=0)$ and $\left(\Delta \mathrm{Q}_{\text {colour }}=0\right)$. We could not predict its lifetime. However if it turns out that proton decay is observed in the future then we could deduce the mass of $Z^{*}$.

Processes which violate A but conserving $L$ or which violate $L$ but conserving A must be strictly forbidden. Proton decay should not be possible if (A-L) is not conserved. We can also predict that neutron antineutron oscillations or neutrinoless double beta decays can not take place in nature. Anyway because neutrino and antineutrino are different neutrinoless double beta decays are strictly forbidden (14). Obtained limits whatever the sensitivity is would reflect only a better and better measurement of zero. If it turns out that a positive signal is univocally detected in the future then there should be something fundamentally wrong in our reasoning.

As long as no microscopic process with violation of $\mathrm{A}$ and $\mathrm{L}$ is observed the only experimental clue leading to speculate that a new interaction beyond the Standard Model must exist is our universe. LHC or collisions of particles and antiparticles in other colliders might provide examples of matter non conservation processes.

\section{Conjecture of a matter creation interaction}

By analogy with weak interaction which requires that TF interpreted as weak charge has to be universally conserved we could conjecture a novel interaction called Matter Creation (MC) defined by matter creation charge $\mathrm{Q}_{\mathrm{MC}}=\mathrm{BAL}$ and a true neutral messenger $Z *(B A L=0, T F=0) . Z *$ could give birth to a baryon lepton pair (or an antibaryon antilepton pair). Interpreted as a charge, BAL has also to be universally conserved. While weak interaction conserves absolutely $\mathrm{Q}, \mathrm{A}, \mathrm{L}$ and $\mathrm{TF}$ (one of these conservation laws is redundant), 
MC conserve only absolutely Q, BAL and TF (one of these conservation laws is redundant). Creation of a pair of oppositely charged baryon-lepton pair or a pair of oppositely charged antibaryon-antilepton pair would allow the creation of a matter asymmetric universe even if $\mathrm{CP}$ is conserved (in the sense that the creation of a baryon-lepton pair is as probable as the creation of an antibaryon-antilepton pair). This hypothesis would explain the strict equality between the proton number and the electron number (due to $\Delta \mathrm{A}=\Delta \mathrm{L}= \pm 1$ and $\Delta \mathrm{THF}=$ $\triangle T L F= \pm 1$ ). In a simplified scenario where we suppose that only protons, antiprotons, electrons and antielectrons are created, the number of protons (or electrons) is a huge number: it is equal to the difference between the number of protons and the number of antiprotons. This number is small compared to the sum of the number of protons and antiprotons. Indeed the quantity $\varepsilon$ defined by:

$$
\varepsilon=\frac{N(p)-N(\bar{p})}{N(p)+N(\bar{p})}
$$

$\varepsilon$ is only nearly zero (the observational value is $1.310^{-9}$ ). We can show the a priori probability that $\varepsilon$ is exactly equal to 0 is nearly $10^{-44}(\mathbf{1 5})$. If all processes of creation of particles or/and antiparticles do conserve $\mathrm{A}$ and $\mathrm{L}$ then $\varepsilon$ should be always equal to zero. During the phase of annihilation one proton annihilated with one antiproton (one electron with one antielectron), only matter particles in excess could remain after the annihilation of all available antiparticles. The quantity $\varepsilon$ which was a tiny number at the moment of the decoupling of MC interaction is at the present time equal to 1 . Indeed the difference $N(p)-N(\bar{p})$ is constant after the decoupling of MC interaction. And this difference is equal to the present number of protons after the disappearance of all antiprotons which annihilated with the same number of protons. This scenario could not account for the precise value of $\varepsilon$. It is rather considered to be incidental and would correspond to initial conditions. Baryogenesis and leptogenesis were indeed an historical event. The non-zero observational value of baryon number was one of the innumerable possibilities corresponding to $2 \mathrm{n}$ processes of matter creation giving with the same probability a pair of baryon-lepton or a pair of antibaryon-antilepton (spontaneous breaking of symmetry). We developed a tossing model to explain why asymmetry was necessarily obtained but the value of the asymmetry was incidental [15].

MC would allow the disintegration of proton into channels verifying $\Delta \mathrm{A}=\Delta \mathrm{L} \neq 0$ but would not allow channels implying $\Delta \mathrm{A} \neq \Delta \mathrm{L}$. Despite gigantic efforts to find out proton decay there is so far no univocal signal. Observation of proton decay if unambiguous should be confronted with our prediction. $Z^{*}$ if it exists is certainly heavier than $Z^{0}(91 \mathrm{GeV})$. But it is 
not necessary that it is as heavy as $\mathrm{X}$ bosons in GUTs $\left(10^{15} \mathrm{GeV}\right)$ and the energy needed to create $Z^{*}$ might be attainable now or in the future in accelerator experiments. The best way to try to create $Z^{*}$ would be to use electron antielectron or proton antiproton collisions. $Z^{*}$ if created would be revealed by the creation of a triquark (uud) and an electron. The signature would be either the detection of an electron together with three jets of quarks (uud) or an electron and a proton.

\section{Charges are universally conserved}

Charge which is the source of an interaction is universally conserved. Thus a quantity which is not always experimentally conserved cannot be considered as a charge (for example strangeness or any other flavour). A and L are apparently conserved in all observed microscopic processes. They thus could be charges and sometimes one speaks of baryonic charge or leptonic charge. However there is so far no evidence that $\mathrm{A}$ is source of baryonic force (4).

The generalized Gell-Mann and Nishijima formula shows that electric charge Q is an algebraic sum of A, L and flavours. Neither A, nor L nor any flavour are charges. Only combination of terms are charges (TF is weak charge, BAL is matter creation charge, $\mathrm{Q}$ is electric charge). Because of this structure of charges it is not only possible to conserve charge by conserving every term of the combination, it is also possible to violate one term of the combination provided that this violation is compensated by the violation of other terms in order to preserve the invariance of the combination.

This possibility explains why weak processes are so abundant and various. Conservation of TF allows weak charged current processes (when individual flavours are not all separately conserved) and weak neutral current processes (when all individual flavours are separately conserved). A given quark can be transformed into a quark of another flavour (an up-like quark into a down-like quark or vice versa with the exchange of $W$ ). Or a charged lepton can be transformed into a neutral lepton or vice versa also by the exchange of $\mathrm{W}$. Neutral current processes via the exchange of $Z$ do conserve all individual flavours. We remark that electromagnetism does conserve all terms relating to $Q$. Strong interaction is independent of $\mathrm{Q}$, the exchange of gluons modify only colour charges, so also does conserve all terms relating to $\mathrm{Q}$.

Conservation of BAL would be very similar to conservation of $\mathrm{TF}$. Interestingly it would permit the simultaneous creation of a complementary pair of baryon-lepton e.g. the creation of only matter particles (or only antimatter particles) via a true neutral messenger $Z^{*}$. (Processes restricted to interaction of the Standard Model are unable to create any imbalance 
between matter and antimatter even if $\mathrm{CP}$ is violated.) It might account for our matter universe. The value of this imbalance would be due to spontaneous breaking of symmetry.

\section{Analogy with CPT invariance}

Before 1957 there was no evidence of violation of any of three reflection symmetries: the invariance of space inversion or parity $\mathrm{P}$; invariance under charge conjugation, $\mathrm{C}$; and time reversal invariance, $\mathrm{T}$. It was at this time admitted tacitly that all these three reflections symmetries were separately conserved. The immediate logical consequence is the invariance of CPT which is the product of these three invariant transformations. In this perspective invariance of CPT does not bring in new information. In reality the CPT theorem was established independently and is believed so far to be absolutely exact. It 'can be derived from only the most fundamental of assumptions, such as the causality of physical events (cause must precede effect), the locality of interactions (instantaneous action at a distance is not possible) and the connection between the spin of particles and the statistics governing their collective behaviour' (16). But the importance of CPT invariance showed up drastically after the fall of parity $(\mathbf{1 7}, \mathbf{1 8})$. In effect one realized that invariance of CPT could also be fulfilled if two of these reflection symmetries were simultaneously violated. Invariance of CPT furnishes then very strict logical constraints. If one transformation is violated then another transformation should be also violated namely the product of two transformations is invariant if both of them are simultaneously invariant or simultaneously violated. There should be a logical implication: if $\mathrm{P}$ is violated either $\mathrm{C}$ or $\mathrm{T}$ has to be violated. It was first thought that in weak interactions violation of parity would be compensated by violation of $\mathrm{C}$ so invariance of $\mathrm{CP}$ would be restored and thus at least $\mathrm{T}$ is invariant. But violation of $\mathrm{CP}$ observed in the decays of $\mathrm{K}$ mesons and $\mathrm{B}$ mesons led logically to the conclusion that $\mathrm{T}$ must be in these cases violated to preserve the invariance of CPT. So none of C or P or T is invariant, the only invariance is CPT. The CPT invariance has important consequences it guarantees that there exists an antiparticle for every particle which has the same half-life, mass and spin and more generally the same arithmetic properties but opposite additive quantum numbers such as $\mathrm{Q}, \mathrm{A}$, $\mathrm{L}$ and flavours and more generally opposite algebraic properties. As far as we know there is no experimental contradiction to these statements.

12. Relation between CPT, $C, P, T$ invariance: Combined and separate reflection symmetries.

Naturally separate $\mathrm{C}, \mathrm{P}$ and $\mathrm{T}$ invariance implies that CPT is invariant. But CPT invariance does not necessarily imply that $\mathrm{C}, \mathrm{P}$ and $\mathrm{T}$ are separately invariant. A transformation may not be invariant under the separate transformations $\mathrm{C}, \mathrm{P}, \mathrm{T}$ or any 
combination of two of these. But if one of them is violated there should be another violation to preserve CPT invariance which is believed to be universally valid. So the decay of $\mathrm{K}_{\mathrm{L}}^{0}$ meson violates both the combined $\mathrm{CP}$ invariance and $\mathrm{T}$ invariance, but not the $\mathrm{CPT}$ invariance. CP violation is directly observed in experiment while violation of $\mathrm{T}$ is only indirectly deduced from invariance of CPT. This meson decays into two conjugate channels $\pi^{+} \mathrm{e}^{-} \bar{v}$ and $\pi^{-} \mathrm{e}^{+} v$ where the latter one is slightly favoured. We remark that this decay is thus not CP invariant in the sense that the two conjugate channels while both possible have not the same probability of occurrence. We could thus define in an absolute way a matter universe. A matter universe is a universe where the charged baryon has the same sign as the lepton of the favoured channel of the decay of $\mathrm{K}_{\mathrm{L}}^{0}$. CP violation in the case of mesons $\mathrm{B}$ is much more important than that of mesons $\mathrm{K}$. One hopes that $\mathrm{CP}$ violation could explain the asymmetry of universe which is a matter universe $(\mathrm{A}>0)$ because it would mean a difference of behaviour between particle (understood as matter particle) and antiparticle (understood as antimatter particle). But the difference in the number of matter particles and the number of antimatter particles is not necessarily due to a difference of behaviour. If we throw $2 \mathrm{n}$ times a coin and do not obtain exactly the same number of faces and tails, we cannot coclude that the coin is not fair. We point out also that the only known cases of $\mathrm{CP}$ violation concern indeed mesons but mesons are neither matter particles $(A>0$ or $\mathrm{L}>0)$ nor antimatter particles $(\mathrm{A}<0$ or $\mathrm{L}<0)$ they are indeed matter neutral particles $(\mathrm{A}=0$ and $\mathrm{L}=0)$ and the antiparticles of mesons are also matter neutral particles $(A=0$ and $L=0)$ (19). The decays of mesons $K$ or $B$ do conserve strictly $\mathrm{A}$ and $\mathrm{L}$ and thus cannot be at the origin of the asymmetry of our universe since the proviso absolute conservation of $\mathrm{Q}$ and violation of $\mathrm{A}$ is not fulfilled. Nevertheless $\mathrm{CP}$ violation (of $\mathrm{K}$ and $\mathrm{B}$ ) without violation of $A$ by itself is and remains a fundamental phenomenon to be studied with scrutiny.

\section{Search of non-zero EDM to test $T$ and $P$ invariance}

Another interesting way to test directly $\mathrm{T}$ invariance and $\mathrm{P}$ invariance is the search of the electric dipole moment (EDM) of the neutron. Existence of non-zero EDM implies automatically that $\mathrm{P}$ and $\mathrm{T}$ are violated. Measurement of EDM is thus a direct test of $\mathrm{T}$ violation and $\mathrm{P}$ violation. Since $\mathrm{P}$ invariance or $\mathrm{T}$ invariance implies that the EDM of the neutron has to be strictly zero any deviation from zero of the EDM means that $\mathrm{T}$ and $\mathrm{P}$ are both violated thus $\mathrm{C}$ is conserved in order to preserve CPT invariance. We remind that historically measurement of the EDM of the neutron (compatible with zero) was considered to be evidence of $\mathrm{P}$ invariance and illustrated conservation of $\mathrm{P}$ in electromagnetism. So far there is no evidence of non-zero value of EDM. Only more and more stringent upper limit on 
the neutron EDM has been obtained setting on possible evidence of direct violation of $\mathrm{T}(\mathbf{2 0})$. The obtained upper limit of the neutron EDM is $2.910^{-26} \mathrm{e}-\mathrm{cm}$. Searches for EDMs could be a good way to reveal new theories of particle physics.

\section{Conclusion}

Paradoxically the only experimental indisputable hint beyond the Standard Model is our neutral universe composed of matter particles. The stage where we observe interactions of the Standard Model is essentially void of antimatter particles. It is in contradiction with interactions of the Standard Model which allow only the creation of a pair of matter antimatter particles. In this context, our universe should be composed half of matter particles, half of antimatter particles as first suggested by Dirac. Since nature cannot realize contradiction a way out would be to postulate a new interaction where $\mathrm{A}$ and $\mathrm{L}$ are no more simultaneously conserved as all interactions of the Standard Model.

Sakharov postulated that CP violation could explain the asymmetry of our universe but violation of baryon number is also necessary. In other words however important it is $\mathrm{CP}$ violation solely cannot explain the asymmetry of the universe. CP violation by itself is certainly a fundamental subject but it could not explain the origin of our material universe if it is not accompanied by the violation of baryon number.

Sakharov introduced the innovative and quite interesting idea of an interaction which would conserve only a combination of $\mathrm{A}$ and $\mathrm{L}$ and proposed that (3A-L) would be the quantity to be conserved supposing that quarks and leptons are on the same footing and predicted that proton decay may occur with a lifetime of $10^{50}$ years.

From observation (material and neutral universe) and from the expression of $\mathrm{Q}$ we deduce that the combination to be conserved is indeed (A-L) supposing that baryons and leptons are on the same footing. A pair of matter particles such as ( $p$ e) out of energy via $Z^{*}$ would be possible (the pair has all charges equal to 0 ). Remarkably theoretical approach inspired by group theory (unified theories like SU(5)) admits also (A-L) invariance as basis but has another constraints.

Considered as a charge, (A-L) has to be universally conserved. It should have very clear-cut predictions: neutrinoless double beta decay and neutron antineutron oscillations should be strictly forbidden. Any positive signal of these processes would be sufficient to disprove our statement. In this framework proton could be instable unlike electron which is absolutely stable since it is the lightest charged particle. There is no sound reason that baryon number and lepton number have to be universally conserved so proton decay is possible even 
if up to now there is no evidence of its decay. If instable the lifetime of proton decay has to be longer than $10^{31}$ to $10^{33}$ years (present experimental limits).

The concept of conservation of combined quantity is fruitful. The conservation or the violation of terms of the combination is logically linked. In the multiplicative case we know that CPT is universally conserved. It may correspond to the conservation of C, P and T (valid for electromagnetism and strong interaction) but it does not forbid the violation of any of these three transformations. Evidence of violation of $\mathrm{P}$ and $\mathrm{C}$ in weak interaction observed in the decay of ${ }^{60} \mathrm{Co}$ and evidence of violation of $\mathrm{CP}$ in $\mathrm{K}$ mesons remind us the importance of experimental verification. Search for of non-zero EDM allows to test $\mathrm{P}$ and $\mathrm{T}$ invariance. A positive signal would be a univocal proof of $\mathrm{P}$ and $\mathrm{T}$ violation. So far there is no evidence of non-zero value of EDM. The obtained upper limit of the neutron EDM is $2.910^{-26} \mathrm{e}-\mathrm{cm}$.

In the additive case conservation of TF is particularly interesting. Weak interaction is associated to $\mathrm{SU}(2)$ group. Flavour changes via the exchange of $\mathrm{W}$ (charged current) but the nature of matter does not change (a quark remains a quark, a lepton remains a lepton). It gives birth to different kinds of processes: purely baryonic processes, purely leptonic processes or semi-leptonic processes. Neutral current via the exchange of $Z$ conserves individual flavours. Its existence is necessary because $Z$ plays the role of neutral element. Electromagnetism is associated to $U(1)$ group, there is only a neutral messenger $\gamma$. The exchange of $\gamma$ does not modify the charge of particles. Strong interaction is associated to $\mathrm{SU}(3)$ group. Colour charges can change with the exchange of gluons. Colour charges are completely independent of electric charge Q. The Standard Model is the union of three interactions electromagnetism, weak interaction and strong interaction considered as the product $U(1) \times S U(2) \times S U(3)$.

We suggest add MC interaction to interactions of the standard model to account for possibly the asymmetry of our universe. All features of the standard model remain naturally valid since the addition of a new interaction MC does not modify the characteristics of particles and interactions of the standard model. So far all observed particle reactions are consistent with the standard model. $Z^{*}$ is as $\gamma$ a neutral messenger. MC interaction which allows proton decay would be associated to U(1) group. Electric charge, weak charge and MC charge are related while colour charges are completely independent of electric charge, weak charge and MC charge. The union of four interactions electromagnetism, MC interaction, weak interaction and strong interaction considered as the product $U(1) \times \mathrm{U}(1) \mathrm{x}$ $\mathrm{SU}(2) \times \mathrm{SU}(3)$ would account for experimental and observational data. Observation of processes violating baryon number would be of great interest to falsify this suggestion. 


\section{References}

1) R. Feynman, The character of physical law (MIT Press 1965) p 63

2) U.C. Tsan, Int.J.Mod.Phys.E16 (2007) 1585

3) U.C. Tsan, Int.J.Mod.Phys.E7 (1998) 405

4 ) A. Franklin, The rise and fall of the fifth force, Ed. American Institute of Physics, New York 1993

5) G. Fraser, The New Physics for the 21st century, Cambridge University Press $p$ 105

5) H. Abele, Progress in Particle and Nuclear physics (2007), doi: 10,1016/j.ppnp.2007.05.002

6) Y.A. Alexandrov, Fundamental properties of the neutron (Clarendon Press Oxford, 1992)

7) H.V. Klapdor-Kleingrothaus et al, Mod.Phys.Lett A, 16(2001)2409

8) H.V. Klapdor-Kleingrothaus et al , Eur.Phys. J.A 12(2001)147

9)H.V. Klapdor-Kleingrothaus et al, Phys.Lett.B 586(2004)198

10)C.E.Aalseth et al, Preprint hep-ex/202018(2002)

11)F. Feruglio, A. Strumia and F. Vissani, Preprint hep-ph/0201291(2002)

12) A.M. Bakalyarov et al, hep-ex/0309016

13) A.D. Sakharov, JETP Lett. 5(1967)24

14) U.C. Tsan, Int.J.Mod.Phys.E11(2003) 439

15) U.C. Tsan, Int.J.Mod.Phys.E7 (1998) 747

16) G.D. Coughlan and J.E. Dodd, The ideas of particle physics, 2nd ed. (Cambridge University Press, 1991), p.48

17)T.D.Lee and C.N.Yang, Phys.Rev.104 (1956) 254

18)C.S.Wu , E.Ambler, R.W.Hayward, D.D.Hoppes and R.P.Hudson, Phys.Rev.105 (1957) 1413

19) U.C. Tsan, Int.J.Mod.Phys.E15(2006) 259

20) C.A. Baker et al, Phys. Rev. Lett. 97, 131801 (2006) 